\title{
Contribuciones de Laplace al desarrollo de la teoría de la probabilidad: en conmemoración de los 200 años de la publicación del "Essai philosophique sur les probabilités" de 1814
}

\author{
Celso Vargas \\ celvargas@itcr.ac.cr \\ Escuela de Ciencias Sociales \\ Instituto Tecnológico de Costa Rica
}

Recibido: Julio 15, 2014

Aceptado: Noviembre 11, 2014

\begin{abstract}
Resumen. En este artículo se analiza la posición que ocupa Laplace en el desarrollo de la teoría clásica de la probabilidad. Se hace en el marco de los 200 años de la publicación del "Essai philosophique sur les probabilités". El artículo se divide en las siguientes secciones: en la primera se introducen algunas de las características de las matemáticas del periodo. En la segunda, se presentan algunos de los desarrollos fundamentales en la teoría de la probabilidad alcanzados durante los siglos XVII y XVIII. Finalmente, presentamos algunas de las principales contribuciones de Laplace. En general, se considera que con Laplace la teoría clásica de la probabilidad adquiere su forma definitiva.
\end{abstract}

Palabras clave: Probabilidad clásica, Laplace, Filosofía de la Probabilidad

Abstract. In this paper the place of Laplace in the development of the classical theory of probability is analyzed. To have a better idea of the context in which the essai was written, some of the main features of the mathematics of that period are introduced. After that, some of the fundamental achievements, during the XVII and XVIII centuries, in probability are presented. Finally, the above mentioned contributions of Laplace to this important subject are briefly discussed. In general, the definitive form of the classical theory of probability is accomplished with this important Laplace's publication.

KeyWords: Classic probability, Laplace, Philosophy of probability 


\subsection{Introducción}

Se reconoce que la formulación precisa del cálculo de probabilidades se debe a Kolmogorov (1933), es decir, presentada de manera axiomática. Una versión en español de esa formalización aparece en Aleksandrov y otros (1982). Sin embargo, cuando se presenta esta formulación ya se habían planteado varias "interpretaciones" diferentes de la probabilidad. La dependencia de la teoría de conjuntos ha hecho que la formulación de Kolmogorov sea criticada fuertemente por diferentes autores, en particular por Popper (1958).

Popper se esfuerza por elaborar una axiomatización de la probabilidad que no dependa y no herede las limitaciones de la teoría de conjuntos, y que pueda expresar su propia concepción de la probabilidad. Pero por otro lado, no todas las interpretaciones cumplen con todos los axiomas de Kolmogorov.

En este momento, las principales formulaciones o interpretaciones de probabilidad son las siguientes: la probabilidad clásica, la probabilidad lógica, la probabilidad subjetiva, las interpretaciones frecuentistas y las interpretaciones propensistas. La discusión de estas distintas interpretaciones son analizados Hájeck, Allan (2011) y en Gillies, Donald (2000). Podemos expresar de manera sintética estas interpretaciones tal y como lo hace Gillies, Donald (2000):

"1. La teoría clásica identifica probabilidad con el grado de creencia racional. Se asume que dada la misma evidencia, todos los seres humanos racionales asignarán el mismo grado de creencia a una hipótesis o predicción.

2. La teoría subjetiva identifica la probabilidad con el grado de creencia de un individuo particular. Aquí no se asume que todos los seres humanos racionales con la misma evidencia tendrán el mismo grado de creencia en una hipótesis o predicción. Se permiten diferencias de opinión.

3. La teoría de la frecuencia define la probabilidad de un resultado como la frecuencia límite con la que el resultado ocurre en una serie larga de eventos similares.

4. La teoría de la propensión, o al menos una de sus versiones, toma la probabilidad como una propensión inherente a un conjunto de condiciones repetibles. Decir que la probabilidad de un resultado particular es p es afirmar que las condiciones repetibles tienen esa propensión de manera que, si estas fueran repetidas un gran número de veces, producirían una frecuencia del resultado cercano a $p^{\prime \prime}$ (Gillies 2000, 1).

Pero en ¿qué consiste la probabilidad clásica? Este será el objeto del presente trabajo. Pero quizá es mejor comenzar con la siguiente pregunta ¿cuál es la relevancia para esta celebración? Esta consiste en que con el Ensayo de Filosofía de la Probabilidad de (1814) culmina el análisis que se hace de la probabilidad durante un siglo y medio. En este sentido, podemos ver este trabajo de Laplace como la formulación, relativamente definitiva, de la probabilidad clásica. Por ello el periodo de nuestro interés se ubica entre la segunda mitad del Siglo XVII y la primera década del siglo XIX. Sin embargo, habrá que hacer referencias obligadas a algunos cambios generales con el fin de comprender mejor la aproximación y los resultados de la probabilidad clásica.

Contribuciones de Laplace al desarrollo de la teoría de la probabilidad: en conmemoración de los 200 años de la publicación del "Essai philosophique sur les probabilités" de 1814. Celso Vargas

Derechos Reservados ( 2015 Revista digital Matemática, Educación e Internet (http://tecdigital.tec.ac.cr/revistamatematica/) 


\subsection{Perspectiva general de la matemática durante este periodo}

En este periodo (segunda mitad del siglo XVII y primera década del siglo XIX) se producen una serie muy importantes de resultados científicos y matemáticos que dejarán su impronta en la manera de entender las probabilidades. Ciencia y matemáticas aparecen tan fuertemente ligadas que los desarrollos en una son también desarrollos en las otras. La renovación en las matemáticas, comenzando por la geometría analítica de Descartes y Fermat (primera mitad del siglo XVII) en la que se logró unificar y tratar bajo un mismo método los tres campos clásicos de la geometría: las figuras que pueden construirse con puntos y líneas rectas (figuras planas), la que resultan de la intersección con un plano (secciones cónicas), las elipses y otras figuras geométricas conocidas como curvas complejas. El método utilizado por Descartes permitió ofrecer soluciones generales y resolver nuevos problemas independientemente del contenido de los objetos. El concepto de magnitud fundamental para la nueva visión mecánica, es un concepto variable que, al utilizarse así, permite aplicar una misma solución en ámbitos en los que se requería un método diferente. No importa que sea un punto, un conjunto discreto o un segmento de recta, todos ellos pueden resolver de la misma manera.

Pero las mayores transformaciones en el campo de la matemática tendrán lugar justo en la segunda mitad de ese mismo siglo: el surgimiento del cálculo diferencial e integral. Como ahora se conoce, éste fue propuesto de manera independiente por Newton y por Leibniz; Newton primero en 1571 y luego Leibniz en 1684. Este nuevo campo de las matemáticas surge directamente relacionado con la solución de problemas físicos dentro de la nueva concepción de explicar todos los fenómenos en términos de tamaño, forma y movimiento. Al respecto, dos perspectivas generales aparecen aquí. Por un lado, la newtoniana que considera la mecánica como la base para la innovación matemática, en el sentido de que el mundo pone de manifiesto que el gran diseñador (Dios) lo construyó siguiendo principios geométricos. En este sentido, los principios mecánicos son también principios geométricos. Newton tiene una gran preferencia por explicaciones geométricas antes que recurrir a hipótesis como la corpuscular. Mejor dicho, vaciló entre una explicación corpuscular para muchos fenómenos o limitarse exclusivamente a una explicación geométrica (Shapiro 2002). Los conceptos newtonianos de fluxión y fluente para designar la integración y la derivación pone de manifiesto el uso mecánico que le da Newton a este nuevo cálculo. Leibniz, por otro lado, prefiere el análisis algebraico de manera que el nuevo cálculo no solo es el fundamento de la física sino también una serie muy amplia de fenómenos como los biológicos, los modales, los éticos y los metafísicos. El uso de una notación algebraica permitió, en el continente, que este nuevo campo de las matemáticas se desarrollara con gran rapidez y de manera robusta.

Contribuyeron de manera significativa, además de Leibniz, los Bernoulli, L’Hospital, Euler, Monge, Lagrange y Laplace para mencionar algunos. Son estos autores los que darán a las matemáticas del periodo las características metafísicas que la distinguen de las matemáticas de los periodos siguientes. Pienso que gran parte de los matemáticos de este periodo son tributarios de algunos de los aspectos de la filosofía de Leibniz, por la claridad que les brindaba a la hora de abordar los nuevos problemas. Son relevantes de la filosofía de Leibniz: a) el principio de continuidad, es decir, de que no hay saltos drásticos en la naturaleza y de que entre el pasado y el presente existe continuidad también, lo mismo que en los otros ámbitos de la naturaleza, también en la cualidad, el movimiento. b) del principio de

Contribuciones de Laplace al desarrollo de la teoría de la probabilidad: en conmemoración de los 200 años de la publicación del "Essai philosophique sur les probabilités" de 1814. Celso Vargas

Derechos Reservados (C) 2015 Revista digital Matemática, Educación e Internet (http://tecdigital.tec.ac.cr/revistamatematica/) 
graduación lineal, es decir, de que la diferencia entre una entidad y la otra es de un atributo, c) el principio de razón suficiente, es decir, de que en la naturaleza nada ocurre sin ninguna causa.

Estos principios permitieron elaborar una visión "metafísica" en la que las matemáticas tenían un importante lugar. Permitió, entre otras cosas, visualizar el continuo como una importante propiedad de las series matemáticas (funciones) de manera que permite afirmar que toda función es continua en todos sus puntos. Como todos sabemos esta visión se mantuvo vigente hasta que Fourier encontró integrales que no cumplían con esta propiedad. Sin embargo, éstas fueron consideradas por Fourier como excepciones, de manera que siguió utilizando este importante principio leibniziano hasta avanzado el siglo XIX (Lakatos, 1981)

Las matemáticas tienen una importante tarea en este contexto, es la ciencia que sistematiza "nuestro conocimiento de la magnitud o cantidad." (Shabel, Lisa 2005, 28). Este concepto de magnitud variará durante el desarrollo de las matemáticas de este periodo, principalmente, en las manos de Newton, Leibniz y de Kant con las que adquiere cada vez mayor abstracción. Sin embargo, lo que es relevante para este trabajo es que, independientemente de estas importantes elaboraciones, las matemáticas de este periodo exhiben tres propiedades importantes: universalidad (la aplicación de un mismo concepto o método a todos los contextos posibles), certeza (está basada en su claridad y distinción) y necesidad (no puede ser de otra manera). De estas tres características deriva su carácter a priori (independiente de su cualquier experiencia), su determinismo (dadas las mismas condiciones, los resultados serán siempre los mismos) y su verdad.

Para finalizar esta breve sección, es de interés presentar de manera sucinta la visión kantiana sobre las propiedades de las matemáticas pues resulta paradigmática de la manera de entender las matemáticas durante este periodo. La revolución copernicana llevada a cabo por Kant, permite captar estas propiedades de las matemáticas de una manera novedosa: dado que nuestro conocimiento es una construcción del sujeto, la percepción del espacio y del tiempo responden a requerimientos matemáticos: las propiedades que atribuimos al espacio expresan las condiciones de toda posible experiencia perceptiva, no existe experiencia sin percepción del tiempo o del espacio. La matemática constituye, entonces, las formas a priori de la percepción. Pero nuestra capacidad esquemática trascendental nos permite construir distintos tipos de generalizaciones, correlaciones y otros procesos de interés matemático. Pero esto podemos hacerlo sin necesidad de "mirar a la experiencia". De esta manera logra una maravillosa síntesis: explicar cómo es posible la universalidad, certeza y necesidad de las matemáticas, y por el otro, porqué es que se aplican con tanto éxito en el conocimiento de nuestro mundo.

\subsection{Desarrollo de la teoría clásica de las probabilidades}

Una de las principales características de la teoría clásica de la probabilidad es que se circunscribe dentro de la concepción matemática anteriormente descrita. De hecho, los autores que la desarrollan son aquellos que participaron también activamente en la construcción de la perspectiva matemática brevemente introducida en la sección anterior. En efecto, en la construcción de esta teoría encontramos a Fermat (1601-1665), Pascal (1623?1662), Huygens (1629?1695), Leibniz (1646-1716), los Bernoulli (fundamental Jakob Bernoulli (1654?1705)), De Moivre (1667-1754), Euler (1707-1783) y Laplace (1749?1827)

Contribuciones de Laplace al desarrollo de la teoría de la probabilidad: en conmemoración de los 200 años de la publicación del "Essai philosophique sur les probabilités" de 1814. Celso Vargas

Derechos Reservados (C 2015 Revista digital Matemática, Educación e Internet (http://tecdigital.tec.ac.cr/revistamatematica/) 
entre los principales autores. Aunque los los estudios actuales sobre probabilidad remontan a Cardano y Galileo, sus contribuciones constituyen formas de resolver algunos problemas específicos, pero no con la sistematicidad con la que encontramos en los autores de nuestro interés. En efecto, para Fermat, Pascal, Huygens, Leibniz, los Bernoulli, Euler y Laplace el estudio de la probabilidad es consistente con los nuevos métodos matemáticos que ellos mismos desarrollaron.

Blaise Pascal (1623?1662) y Pierre de Fermat (1601-1665) abordaron un problema propuesto por el famoso apostador Chevalier de Méré conocido como el "reparto de apuestas". Consiste en determinar qué porcentaje de la apuesta le corresponde a cada jugador (para el caso de dos) en caso de que decidan no continuar jugando y tomando en consideración lo que han ganado. Por ejemplo, si han apostado para ver quién gana en 2 , o en 4 , o en 6 , etc., tiradas de dados, y deciden concluir el juego antes de la segunda jugada, la cuarta, y así sucesivamente. La aproximación que ambos matemáticos hacen es combinatoria: se estiman las combinaciones posibles y se determina el porcentaje correspondiente a lo asegurado en las jugadas anteriores y la distribución del remanente del total para las jugadas que restarían por realizar. Pascal propone dos soluciones generales (entre los años de 1654-1660) ambas basadas en combinaciones, para un número arbitrario de tiradas entre dos jugadores, y para el caso específico del lanzamiento de dados. Divide las tiradas entre aquellas impares y las pares correspondientes a cada jugador. Por ejemplo, si el juego se establece en 5 jugadas, entonces, tendríamos 10 lanzamientos en total. Pascal los separa entre números pares e impares. El producto de los impares es el numerador y el producto de los pares el dominador. De esta manera dice Pascal, lo que le corresponde a un jugador sería el producto de los impares dividido por el producto de los pares hasta la jugada realizada más la probabilidad de ganar la siguiente jugada. Adicionalmente, Pascal utiliza el triángulo aritmético para determinar la probabilidad de obtener un resultado dentro de un número determinado de alternativas. Por ejemplo, si se trata de sacar un número premiado de una tómbola de entre cinco disponibles, entonces, se utiliza la secuencia 1510105 1, es decir, 1 opción de no obtener el número premiado, 5 de obtener uno, 10 de obtener dos números premiados, 10 de obtener 3 números premiados, 5 de obtener cuatro números premiados y 1 de obtener los cinco números premiados. De un total de 32 combinaciones (correspondencia Pascal-Fermat).

El siguiente importante paso en el desarrollo de la teoría clásica de la probabilidad proviene de Huygens al presentar un resultado más general que el de Pascal para un número mayor de jugadores, específicamente, tres. Sin embargo, lo hizo de manera independiente de Pascal y Fermat. Lo introduce en su correspondencia con Mylon, Carcavy y Shooten durante los años de 1656 e inicios de 1657.

Leibniz también se ocupó del tema de las probabilidades en dos momentos de su carrera intelectual. Primero en su ensayo "Estimación de la Incertidumbre" escrito en 1678 en el que presenta un pequeño sistema axiomático para formalizar los conceptos de "esperanza" (probabilidad de ganar) y "miedo" (probabilidad de perder) en un juego justo para un número cualquiera de jugadores. En un segundo momento en su correspondencia con Jakob, quien introdujo lo que se ha dado en llamar "Ley de los grandes números". Esta correspondencia tiene lugar durante los años 1703-1704. Como recordara Jakob muere relativamente joven a los 50 años y meses, en 1705. En su correspondencia Jakob siempre señala problemas de salud que le impiden avanzar en el trabajo como el deseara.

Tanto en la correspondencia con Leibniz como en su obra Ars Conjectandi, se observa un importante giro en la reflexión sobre probabilidad y a los ámbitos a los que puede aplicarse. En efecto, tal y como lo señala Glenn Shafer (2005) el abordaje sobre probabilidad en los autores mencionados anteriormente, incluyendo a Leibniz, tienen que ver mucho más con el concepto de "justeza" aplicado en el análisis 
de varios fenómenos relacionados con el juego justo. Será entonces Bernoulli el que tratará de extender el análisis a otros ámbitos no considerados hasta el momento. Leibniz no encuentra convincente esta extensión pues claramente no se reúnen las condiciones de "justeza" que debe caracterizar esta aproximación.

Para tener una mejor idea de lo anterior, veamos algunos de los cambios introducidos por Jakob Bernoulli. Para ello nos guiaremos tanto por la correspondencia con Leibniz como por el trabajo realizado por Glenn Shafer y citado anteriormente. En el tiempo en que Jakob Bernoulli escribe su Ars Conjectandi, a la edad de 30 años (1684), pero que es publicada en 1713, había dos ámbitos separados que tenían que ver con probabilidad, aunque no se utilizada todavía este término, a saber: los juegos de azar, a los cuales se aplica muy bien lo encontrado por Huygens, Leibniz, Pascal y Fermat, y el otro, los problemas políticos, judiciales, personales y de negocios a los que se les aplicaban máximas para "pesar probabilidades". Este último ámbito era completamente excluido del estudio de la teoría matemática del juego justo, debido a la dificultad de expresarlo en términos combinatorios o con distribuciones a priori. El encontrar una misma forma para el tratamiento de ambos dominios, será una de las grandes contribuciones de Bernoulli. Esto no se puede hacer sin cambiar el concepto de probabilidad que había sido manejado hasta el momento por el nuevo: la probabilidad "como parte de la certeza?.'la probabilidad es un grado de certeza, y difiere de la certeza como una parte del todo" (Shafer 2005, 7). Así pues, entender la probabilidad de este modo permite, entonces, considerar tanto los juegos de azar como el "peso de probabilidades" como dos formas que expresan grados de certeza. Esta segunda rama de estudio de las probabilidades fue desarrollada posteriormente por varios matemáticos incluyendo Deparcieux, Kersseboom, Wargentin, Dupré de Saint-Maure y Simpson (Laplace, 1814, 189).

La ley de los números grandes (o teorema de los grandes números) es otra de las grandes contribuciones de Jacob Bernoulli al desarrollo de la teoría de la probabilidad. Proporciona un importante método para determinar el comportamiento de una secuencia de eventos conocidos ("observaciones" las denomina Bernoulli) cuando esta forma parte de un número muy grandes de estos eventos. Es entonces, un método para encontrar distribuciones de eventos mostrando que estos convergen a un cierto valor cuando el número de eventos tiende al infinito.

Este cambio en la concepción de la probabilidad comienza a observarse, señala Shafer, en el trabajo de Abraham De Moivre de 1718, cinco años después de la publicación del Ars Conjectandi. De esta manera, comienza a imponerse, y Abraham De Moivre juega un papel importante, la teoría según la cual, probabilidad concierne con los siguientes tres elementos: a) la probabilidad como la razón de casos igualmente probables (perspectiva clásica); b) reglas para asignar y valorar expectativas y c) reglas para combinar probabilidades. Este tercer aspecto es una gran novedad en ese momento, pues hasta el momento no se había pensado en distinguir entre "elementos simples" o "eventos simples" y aquellos que pueden obtenerse por combinación siguiendo determinadas reglas.

Finalmente, para concluir esta sección haremos una breve referencia a Euler en relación con el desarrollo de la teoría clásica de la probabilidad. Tal y como lo han señalado Shafer (2005) y Sheynin (2013), Bernoulli no presentó una demostración formal de su teorema de los grandes números. Esto tendrá que esperar hasta desarrollos posteriores para la estimación de inecuaciones intermedias requeridas para el cálculo. Pero Euler llegó a un resultado similar al de Bernoulli en su trabajo sobre funciones cotangentes, de manera más general (Gautschi, Walter 2008). Adicionalmente, encontró otras series armónicas que son utilizadas en la estimación de distribuciones de frecuencias. En este sentido, una importante contribución de Euler fue haber integrado la teoría de probabilidades a un ámbito matemático más general, es decir, como parte de la teoría sobre series convergentes. 


\subsection{Aproximación y contribuciones de Laplace a la teoría de las}

\section{probabilidades}

El trabajo de sistematización de la probabilidad que lleva a cabo Laplace se hace teniendo como telón de fondo los desarrollos que hemos esbozado brevemente. Pero más que eso, los elementos principales señalados en nuestra breve historia son los articuladores de su teoría.

(a) Adopta la posición de Bernoulli de que la probabilidad está relacionada con la certeza, o mejor, como lo indica Laplace, "La probabilidad es relativa, en parte a esta ignorancia, en parte a nuestro conocimiento. Conocemos que de tres o de un mayor número de eventos que un único evento debe suceder; pero nada nos induce a creer que uno de ellos sucederá y no los otros. En este estado de indecisión es imposible para nosotros anunciar su ocurrencia con certeza. Es sin embargo probable que uno de estos eventos, escogido a voluntad, no suceda porque vemos varias causas igualmente posible que excluyen su ocurrencia, mientras a solo uno de estos le favorece" (Laplace 1814, 6). En este sentido, ignoramos cuál de estos eventos sucederá, pero del análisis de sus causas o condiciones, podemos establecer una distribución de probabilidad de su ocurrencia. ¿Pero en qué consiste la certeza probabilística? En el penúltimo capítulo de la obra que comentamos, Laplace ofrece un interesante análisis sobre la certeza en la que compara tres o quizá cuatro métodos generales: la deducción (demostraciones), la inducción, la analogía y la probabilidad. Sin duda dice, que la inducción y la analogía han sido los más fecundos en promover el avance de las ciencias. Sin embargo, el método de las probabilidades, que comparte la certeza de las ciencias exactas, pero el mismo tiempo, no se pronuncia sobre las causas, constituye un valioso instrumento para complementar la investigación inductiva y analógica, dando criterios adicionales de razonabilidad para las hipótesis que se planteen. En este sentido, la certeza probabilística adquiere su importante lugar en el ámbito de las ciencias.

(b) Nada ocurre sin ninguna causa. Este principio Leibniziano (principio de razón suficiente) es citado por Laplace como axioma para la investigación científica, la vida cotidiana y la probabilidad. Lo que sucede con eventos probabilísticos es que no tenemos suficientes elementos para determinar la causa de la ocurrencia del evento, entre varias posibles para un conjunto de eventos. Si pudiéramos conocer cuál de las causas posibles se presenta, podríamos predecir igualmente cuál de los eventos posibles sucederá. La insuficiencia de conocimiento está a la base de la teoría de la probabilidad. Pero una vez que las causas sean identificadas y diferenciadas según eventos, podremos atribuir con necesidad la ocurrencia de tales eventos. Esto es conocido como el demonio de Laplace.

(c) La teoría de la probabilidad de Laplace ya incorpora el redimensionamiento aludido cuando presentamos las contribuciones de Bernoulli en el sentido de que los juegos de azar (juegos justos) son solo uno de los ámbitos a los que se aplica esta teoría. En este aspecto Laplace señala: "Presento aquí sin ayuda del análisis los principios y los resultados generales de esta teoría, aplicándolos a los más importantes asuntos de la vida, los cuales son en verdad en su mayor

Contribuciones de Laplace al desarrollo de la teoría de la probabilidad: en conmemoración de los 200 años de la publicación del "Essai philosophique sur les probabilités" de 1814. Celso Vargas

Derechos Reservados @ 2015 Revista digital Matemática, Educación e Internet (http://tecdigital.tec.ac.cr/revistamatematica/) 
parte problemas de probabilidad"( pág. 12). En este sentido, las matemáticas determinísticas, es decir, aquellas en las que se garantiza la certeza, constituyen lo que podríamos llamar "un caso particular" de las teoría de las probabilidades. En el "Essai philosophique sur les probabilités", Laplace dedica, la segunda parte, y la más extensa a analizar las aplicaciones de la teoría de la probabilidad a campos tan diversos como: la filosofía natural, los juegos de azar, aquellos ámbitos en los que existen "desigualdades desconocidas" en casos en los que se supone que son iguales (por ejemplo, casos en los que un dado esté desbalanceado), su uso en las ciencias sociales, en el análisis de testimonios, en los juicios en tribunales de justicia, en estudios epidemiológicos y la duración de los matrimonios y decisiones en asambleas. El capítulo XVIII con el que finaliza el libro, adelanta la siguiente sentencia: "Es digno de observar que una ciencia, que comenzara considerando los juegos de azar, fuera elevada al rango de los más importantes temas del conocimiento humano"( página 195)

(d) La diferencia entre la probabilidad de eventos simples y la estimación de la probabilidad en series complejas de eventos, es decir, la combinación de probabilidades. En este sentido, las contribuciones de Abraham De Moivre de 1718 formaron a ser parte estructural de la propuesta de Laplace. La teoría de la probabilidad es de naturaleza combinatoria.

Lo anterior ha llevado algunos autores a afirmar que Laplace contribuyó muy poco al desarrollo de la teoría de la probabilidad y que fue más bien un sistematizador de los desarrollos realizados durante algo más de 150 años. No vamos a revisar esta tesis aun cuando claramente se observa esta incorporación. Laplace mismo en el último capítulo titulado "Nota histórica relacionada con el cálculo de probabilidades" (capítulo XVIII) es muy generoso en reconocer la contribución de sus antecesores en este desarrollo. Esto pone de manifiesto el espíritu disciplinario y estudios de Laplace.

Tal y como lo expresa Laplace, entonces, dadas determinadas probabilidades para eventos singulares o individuales (denominadas por Laplace posibilidades), la tarea de la teoría de probabilidad es proporcionar las reglas generales para combinar eventos de cualquier longitud y estimar la esperanza; esta última definida como "la ventaja que uno tiene de obtener ciertos beneficios suponiendo que estos son solamente probables" (página 20). Laplace propone un total 10 reglas para series de eventos probabilísticos, distribuidos de la siguiente manera: 7 reglas para estimación de la probabilidad de eventos y 3 para el concepto de esperanza.

Dado un número de posibilidades igualmente probables (por ejemplo, del 1 al 6 en un dado), regla 1, la probabilidad de ocurrencia de uno de estos eventos (digamos 6) es el número de casos favorables, sobre el número total de eventos posibles, en este caso, 1/6. De acuerdo con la regla segunda, la estimación de la distribución de probabilidad se hace de manera combinatoria. Tal y como lo indicamos esta tercera regla es fundamental para la teoría clásica de la probabilidad. De acuerdo con la regla tercera, si los eventos son independientes, entonces, la probabilidad combinada de la ocurrencia de cierta o ciertas secuencias de interés, es el producto de sus probabilidades individuales o la de los posibles. La cuarta regla establece cómo se estima la probabilidad en aquellos casos en los que hay eventos dependientes. El cálculo de la probabilidad es el mismo que el anterior, es decir, el producto de la probabilidad del primero por el del segundo, con la condición de que si se da el primero, tendrá que darle también el segundo. La quinta regla nos indica que en la estimación de la probabilidad de eventos, no es relevante la consideración del pasado en la estimación de los eventos bajo consideración. La regla sexta tiene que ver con la manera en la que debemos calcular la probabilidad de las causas a partir de los efectos, es decir, en aquellos casos en los que tenemos a priori fuertes razones para pensar que ocurrió con una 
causa. El ejemplo que da Laplace es el de la palabra "Constantinopla". La probabilidad de que este evento ocurra no al azar sino por una causa es considerablemente alta.

Este tipo de eventos extraordinarios (es decir, que son combinatorios) deben ser valorados de manera diferente a aquellos en los que a priori tenemos razones para considerarlos como al azar. De acuerdo con Laplace este grupo es muy pequeño en comparación con el combinatorio. En este caso, para estimar la probabilidad lo hacemos de la siguiente manera: "la fracción cuyo numerador es la probabilidad del evento que resulta de esta causa y cuyo denominador es la suma de las probabilidades similares relativas a todas las causas" (página 15). Finalmente, la séptima regla, tiene que ver con aquellos casos en los que hay una variación en las condiciones actuales con lo cual cambiarían las probabilidades de un evento futuro (es decir, la predicción de eventos). El autor analiza varios casos, por ejemplo, la probabilidad de que el sol salga mañana. Pero otro ejemplo podría ser el siguiente (presentado por el mismo Laplace): En tres urnas A, B y C, una de ellas contiene una bola blanca, la probabilidad de que esté en cualquiera de ellas es de $1 / 3$. Supongamos que yo apuesto a que está en B. Claramente, la probabilidad de ganar es de $1 / 3$. Pero si, obtengo la información de que no está en A, cuál es la probabilidad que tengo ahora de que esté en B. De acuerdo con esta regla, la probabilidad de este evento se distribuye, de manera que, la probabilidad de que esté en B es $1 / 2$ en la nueva situación. Como se sabe este ha sido uno de los puntos de discrepancias más importantes con interpretación subjetiva de la probabilidad dentro de la cual no es válido llevar a cabo tal distribución.

Las tres reglas presentadas por Laplace para estimar la esperanza, definida ésta como "la ventaja que uno tiene de obtener ciertos beneficios suponiendo que estos son solamente probables" (página 20), son las siguientes:

La primera establece que el beneficio o ventaja se obtiene considerando el producto de la probabilidad correspondiente a cada uno de los eventos ganados por el beneficio asociado con la ocurrencia del evento. La segunda regla establece que en la estimación del beneficio se debe considerar la suma de los beneficios obtenidos por la regla primera pero sustrayendo la suma de lo perdido en cada uno de los eventos desfavorables. Finalmente, la regla tercera establece que "el valor relativo a una suma infinitamente pequeña es igual al valor absoluta de dicha suma dividido por el beneficio total de la persona interesada" (página 23). Tal y como señala Laplace, la aplicación de esta regla supone que el beneficio a obtener por cualquier persona en cualquier momento es diferente de cero. De esta manera concluimos la presentación de las reglas. Como se observa estas reglas se aplican para probabilidades entendidas como combinaciones excluyendo los eventos extraordinarios.

Para finalizar nuestra breve presentación de Laplace, queremos presentar los tres métodos generales establecidos por Laplace para obtener las distribuciones de probabilidad en un marco de naturaleza combinatoria. Claramente, desde esta perspectiva los tres métodos son equivalentes.

(a) A priori. Cuando atendemos a la naturaleza de los eventos a considerar y determinamos que, por ejemplo, un dado es homogéneo y por lo tanto, cualquiera de los números puede ocurrir con la misma probabilidad. Para la combinación de probabilidad a partir de los posibles, seguimos las reglas definidas anteriormente

(b) A posteriori. Se presenta cuando realizamos determinamos experimentos para establecer las posibilidades y las distribuciones de probabilidad para la repetición de un evento dentro de una secuencia. Dicha distribución de valores nos puede convencer de que el dado, en nuestro ejemplo, 
está cargado o que, por el contrario es homogéneo. En ambos casos podemos afirmar que la probabilidad de que ocurra un 6 se aproxima a $1 / 6$ si el dado es homogéneo, menor a $1 / 6$ si está cargado en desventaja del 6 y que es mayor a $1 / 6$ en caso de que la carga favorezca la ocurrencia del 6. A posteriori lo que aprendemos es que hay una causa identificable en aquellos casos en los que observemos un dado cargado a favor o en contra de una determinada ocurrencia

(c) Buscando las razones. Difiere del caso anterior en el tanto no requerimos realizar experimentos para establecer las posibilidades, sino que analizando las razones que tenemos para determinar la ocurrencia de un evento podemos concluir, por ejemplo, que si los jugadores de dados se encuentran en igualdad de condiciones y el dado es homogéneo, entonces, la probabilidad de la ocurrencia de un 6 es 1/6. Buscando las razones para que ocurra un evento nos permite igualmente encontrar las explicaciones para las secuencias de eventos extraordinarios

\subsection{Conclusión}

A modo de conclusión, la influencia del "Essai philosophique sur les probabilités" de 1814 de Laplace ha sido muy importante ya que presenta, como hemos visto, una sistematización de los desarrollos obtenidos durante un siglo y medio, dándole forma a lo que se conoce como "teoría clásica de la probabilidad". En efecto, cuando se habla de la teoría clásica se utiliza como sinónimo de Laplace, salvo las contribuciones que realizó Gauss sobre probabilidad, incluyendo su muy ampliamente utilizada campana de Gauss, cuya aplicación en la ciencia y en la comprensión de fenómenos sociales ha mostrado un gran poder explicativo. Estas distribuciones de probabilidad fueron desarrolladas por Gauss en 1809, cinco años antes de la publicación del Ensayo sobre Filosofía de la Probabilidad. Laplace en el capítulo XVIII hace una muy breve referencia a Gauss pero no desarrolla el tema de las distribuciones normales, sino más bien a la urgencia de estimar algún margen de error cuando existen condiciones desconocidas.

\section{Bibliografía}

[1] Aleksandrov y otros (1982). "La matemática: su Contenido, Método y Significado." Alianza Editorial, España. 3 volúmenes.

[2] Bernoulli, Jakob. "Pars Quarta tradens Usum \& Applicationem Praecedentis Doctrinae in Civilibus, Moralibus E Oeconomicis" http://www. sheynin.de/download/bernoulli.pdf

[3] Gautschi, Walter (2008). "Leonhard Euler: His Life, the Man, and His Works". SIAM REVIEW Vol. 50, No. 1, pp. 3?33.

[4] Gillies, Donald (2000)."Philosophical Theories of Probability". Editorial Routledge, London.

[5] Hájek, Alan (2011). "Interpretations of Probability". Entrada: Standford Encyclopedia of Philosophy http://plato.stanford.edu/entries/probability-interpret/

[6] Lakatos, Imre (1981).Matemáticas, Ciencia y Epistemología. Alianza Editorial, España.

[7] Leibniz, W. (1678). "Estimación de la Incertidumbre" en Echevarría, Javier editor 1999, Gottfried Wilhelm Leibniz, Escritos Metodológicos y Epistemológicos, Escritos Filosóficos, Lógico-Matemáticos,

Contribuciones de Laplace al desarrollo de la teoría de la probabilidad: en conmemoración de los 200 años de la publicación del "Essai philosophique sur les probabilités" de 1814 . Celso Vargas

Derechos Reservados (C 2015 Revista digital Matemática, Educación e Internet (http://tecdigital.tec.ac.cr/revistamatematica/) 
Sobre Máquinas y Ciencias Físico-Naturales, Jurídicos, Políticos y Sociales, Teológicos y Religiosos. Editorial Gredos, España.

[8] Leibniz y Bernoulli. "Correspondence Regarding The Art Of Conjecturing." http://cerebro.xu . edu/math/Sources/JakobBernoulli/jakob

[9] Popper, Karl (1958). "La Lógica de la Investigación Científica." Edición española, Editorial Tecnós, España, 1982.

[10] Shabel, Lisa (2005). "Apriority and application: Philosophy of Mathematics in the Modern Period". En Shapiro Stewart editor (2005) The Oxford Handbook of Philosophy of Mathematics and Logic. Oxford University Press.

[11] Shafer, Glenn (2005). "The Significance of Jacob Bernoulli?s Ars Conjectandi for the Philosophy of Probability Today" http://www.matematica.ciens.ucv.ve/modelos/Descargas/article55.pdf

[12] Shapiro, Allan E. (2002). "Newton's optics and atomism." En Cohen, Bernard y Smith, George, editores, (2002) Cambridge Companion to Newton. Cambridge University Press. Pp.227-255.

[13] Sin autor (s.f) "Fermat And Pascal On Probability" http://www.york.ac.uk/depts/maths/ histstat/pascal.pdf

[14] Sin autor (s.f) "Correspondence of Huygens Regarding Games of Chance. " http://cerebro.xu. edu/math/Sources/Huygens/sources/correspondence $\% 20$ of $\% 201656$.pdf

Contribuciones de Laplace al desarrollo de la teoría de la probabilidad: en conmemoración de los 200 años de la publicación del "Essai philosophique sur les probabilités" de 1814 . Celso Vargas

Derechos Reservados (C 2015 Revista digital Matemática, Educación e Internet (http://tecdigital.tec.ac.cr/revistamatematica/) 